\title{
The effects of hemodynamics on the inner layers of the aortic wall in patients with a bicuspid aortic valve
} \author{
Deborah IE Schoor ${ }^{8}$, Robert JM Klautz ${ }^{1}$, Robert E Poelmann ${ }^{8,9}$ and Adriana C Gittenberger-de Groot ${ }^{9}$ \\ ${ }^{1}$ Department of Cardiothoracic Surgery, Leiden University Medical Center, Leiden, the Netherlands \\ ${ }^{2}$ Department of Anatomy and Embryology, Leiden University Medical Center, Leiden, the Netherlands \\ ${ }^{3}$ Department of Cardiovascular Surgery, University Heart Center Hamburg, Germany \\ ${ }^{4}$ Department of Cardiac Surgery, Central Hospital Bad Berka, Bad Berka, Germany \\ ${ }^{5}$ Department of Molecular Cell Biology, Leiden University Medical Center, Leiden, the Netherlands \\ ${ }^{6}$ Department of Vascular Surgery, Leiden University Medical Center, Leiden, the Netherlands \\ ${ }^{7}$ Department of Medical Statistics, Leiden University Medical Center, Leiden, the Netherlands \\ ${ }^{8}$ Institute of Biology, Animal Sciences and Health, Leiden University, Leiden, the Netherlands \\ ${ }^{9}$ Department of Cardiology, Leiden University Medical Center, Leiden, the Netherlands
}

Nimrat Grewal ${ }^{1,2}$, Evaldas Girdauskas ${ }^{3,4}$, Marco C DeRuiter $^{2}$, Marie-José Goumans ${ }^{5}$,Jan H Lindeman ${ }^{6}$, Kushtrim Disha ${ }^{3}$, Ron Wolterbeek ${ }^{7}$,

\begin{abstract}
Background: A bicuspid aortic valve (BAV) is the most common congenital cardiac malformation and is associated with ascending aortic dilation in $60-80 \%$ of patients. Structural differences in aortic wall architecture have been noted between patients with BAV and a tricuspid aortic valve (TAV). The purpose of this study was to analyse a possible correlation between hemodynamics and vessel wall structure in aortopathy in BAV patients.

Methods: BAV ( $n=36)$ and tricuspid aortic valve (TAV) ( $n=17)$ patients undergoing aortic valve replacement underwent pre-operative flow MRI assessment to detect the area of maximal flow-induced stress in the proximal aorta. Based on these MRI data paired aortic wall samples (i.e. area of maximal jet impact (jet sample) and the opposite aortic wall (non-jet sample)) were collected during surgery. The jet and non-jet sample were graded for seven histopathologic features, referred to as pathology score.

Results: Comparing the jet and non-jet samples in both BAV and TAV, regions of maximal jet impact did not show any difference in the pathology score in the adventitia, middle and outer media even if corrected for aortic stenosis/ regurgitation, aortic dilation and raphe position. In the jet samples, the inner media however showed loss of actin expression in both BAV $(\mathrm{p}<0.0001)$ and the TAV $(\mathrm{p}=0.0074)$ and the intima thickness was significantly enlarged in both patient groups $(\mathrm{BAV}$ $\mathrm{p}=0,0005, \mathrm{TAV} \mathrm{p}=0,0041)$.
\end{abstract}

Conclusions: On the jet side an activation of the inner layers of the aortic wall is seen in all patients of both BAV and TAV groups.

\section{Introduction}

Bicuspid aortic valves (BAVs) are associated with an increased risk for aortic dilation and dissection [1,2]. Many studies have focused on the pathogenesis of aortopathy in BAV patients, which can roughly be divided into two categories advocating either genetic or hemodynamic causal factors. Structural differences in aortic wall histopathology have been shown between patients with BAV and a tricuspid aortic valve (TAV) [3-6]. The ascending aortic wall in all BAV patients is characterized by an immaturity of the vascular smooth muscle cells (VSMC) [3] and less ageing histopathology features, such as inflammation and cystic medial degeneration (CMD) [7]. Structural differences of the aortic wall between both valve types have recently been accepted in the clarification statement from the American College of Cardiology/ American Heart Association, stating that histologic properties of the ascending aorta between BAV and TAV differ, raising the possibility that the aortic wall might be more vulnerable for dilation in BAV patients [8]. Several attempts have been made to detect molecular biological factors to identify BAV patients with an apparent non-dilated aorta but possibly an increased susceptibility for aortic complications $[4,9]$.

Also the role of valve related hemodynamics in the development of aortic complications in BAV is being studied [10-12]. Flow-sensitive cardiac magnetic resonance imaging (CMR) with full volumetric coverage of the ascending aorta (4-dimensional 4D flow CMR) can visualize and measure aortic 3-dimensional blood flow patterns, such as flow jets, vortices, and helical flow. It has been shown that even

Correspondence to: Adriana Gittenberger- de Groot, Dept. of Cardiology, Leiden University Medical Center Postal zone: S-5-24, P.O. Box 9600, 2300 RC Leiden, The Netherlands, Tel: +31-71-526 3704(9306); Fax: +31-71-526 6809; E-mail: acgitten@lumc.nl

Key words: bicuspid aortic valve, shear stress, aortopathy, histopathology, tricuspid aortic valve

Received: September 22, 2017; Accepted: October 20, 2017; Published: October 23,2017 
non-stenotic or regurgitant BAVs are still associated with disturbed flow patterns in the ascending aorta, with regional increases in wall shear stress $[13,14]$.The question, however, remains whether the impact of jet on the ascending aortic wall is sufficient to explain the observed structural differences of the aortic wall between BAV and TAV. It is also still unclear whether the hemodynamic effects on the aortic wall can be related to dilation of the ascending aorta, the raphe position in the valve leaflets or stenosis or insufficiency of the aortic valve. In search of a solution, we studied the effect of hemodynamics on the histopathology of ascending aortic wall in both BAV and TAV patients. We measured the area of maximal jet impact by flow magnetic resonance imaging and analyzed the histopathology characteristics in paired jet and non-jet-samples of BAV and TAV aortic wall tissue.

\section{Material and methods}

\section{Ethical approval}

Sample collection and handling was carried out according to the official guidelines of the Medical Ethical Committee of the Central Hospital Bad Berka. All patients gave written informed consent.

\section{Study population and preoperative cardiac phase-contrast cine magnetic resonance imaging examination}

All consecutive patients with a BAV or a TAV with stenosis or regurgitation undergoing aortic valve replacement (AVR) with or without concomitant proximal aortic replacement at the Central Hospital, Bad Berka, Germany from January 2012 through February 2014 were evaluated prospectively. A total of $36 \mathrm{BAV}$ patients (mean age $55.8 \pm 8.7$ years, $72 \%$ male) and 17 TAV (mean age $60 \pm 8.9$ years, $82 \%$ male) were included in the study. The BAV and TAV patients were subdivided in 6 groups on the basis of aortic dilation, aortic stenosis or regurgitation and raphe position (for BAV patients). The morphology and function of the aortic valve was assessed preoperative by echocardiography and cardiac phase-contrast cine magnetic resonance imaging (MRI) in all patients. An aortic valve was considered bicuspid if two-dimensional (2D) short-axis imaging of the aortic valve demonstrated the existence of only two commissures delimiting two aortic valve cusps. The final decision regarding the bicuspidy of aortic valve was however made based on the intraoperative description of valve anatomy by the surgeon, i.e. the existence of three normal commissures for TAV and two normal commissures for BAV. Aortic valve stenosis and regurgitation was defined according to guidelines [15].

The diameter of the proximal aorta was measured preoperatively by means of transthoracic echocardiography and MRI. Dilation was defined by reaching an ascending aortic wall diameter of $45 \mathrm{~mm}$ or more [8]. Patient characteristics are summarized in Table 1.

All patients underwent cardiac phase-contrast cine MRI examination preoperatively to detect the area of maximal jet impact in the proximal ascending aorta. A single non-contrast cardiac MRI (Avanto 1,5T scanner; Siemens, Erlangen, Germany) which included structural, functional and phase-velocity-encoded imaging of the left ventricular outflow tract (LVOT) and the proximal aorta was performed as has earlier been described [16]. Figure 1 shows the location of the jet and the the angle at which the flow jet hits the aortic wall in the flow velocity-encoded window.

\section{Tissue samples}

Based on preoperative MRI analysis, two aortic specimens were collected from the aortotomy incision in patients who underwent isolated AVR only and from intraoperatively excised aortic tissue in the patients who required simultaneous ascending aortic replacement. The first aortic specimen obtained from the area of maximal jet impact as identified by MRI analysis was called the 'jet-sample'. The second sample was collected from the opposite aortic wall and is referred to as the 'non-jet sample'. Both samples were fixed in $4.5 \% \mathrm{pH}$ buffered formalin and embedded in paraffin by the Institute of Pathology, University Hospital Jena, Germany. In the Leiden University Medical Center, transverse sections were mounted on precoated Starfrost slides (Klinipath BV, Duiven, The Netherlands).

\section{Histopathologic parameters and analysis}

Transverse sections ( $5 \mu \mathrm{m})$ were stained with haematoxylin-eosin, resorcin fuchsin, and for alpha smooth muscle actin (aSMA) and smooth muscle 22 alpha (SM22a). The staining protocols have been described in detail in a previous paper (3) of our group. MOVAT pentachrome staining was performed on $4 \mu \mathrm{m}$ sections, the staining protocol was used as described before [17].

A pathology score was developed based on the sum score $[7,16]$ and the recently published aortic consensus paper statement on surgical pathology of the aorta [18]. We selected histopathologic parameters, which were, if applicable, graded (semi-) quantitatively in the tunica intima, media and adventitia. The following parameters were studied 1) Intimal architecture, including endothelial cell and subendothelial layer structure and the absolute intimal thickness (the distance between the endothelial layer and the first major internal elastic lamellae, excluding atherosclerotic areas) in $\mu \mathrm{m}$. 2) Intimal atherosclerosis indexed from 0 (none), 2 (mild), 4 (moderate) to 6 (severe). 3) Medial VSMC differentiation, based on the expression of the differentiated VSMC marker SM22a (19), indexed from 0 (no expression in VSMCs), 2 (expression in less than one-third of all VSMCs), 4 (expression in two-third of all VSMCs) to 6 (expression in more than two-third of all VSMCs), 4) Medial elastic fiber degradation (EFD) indexed from 0 (none), 2 (mild), 4 (moderate) to 6 (severe), 5) Medial mucoid extracellular matrix accumulation (MEMA) indexed from 0 (none), 2 (mild), 4 (moderate) to 6 (severe). 6) Loss of VSMC nuclei in the tunica media (CMD) indexed from 0 (none), 2 (mild), 4 (moderate) to 6 (severe) and 7) Adventitial inflammation (presence of a cellular infiltrate in the adventitia), indexed from 0 (no inflammatory cells), 2 (a few cells), 4 (groups of cells) to 6 (large clusters of cells).

We performed an additional analysis on the expression of aSMA in the inner media indexed from 0 (expression in less than one-third of all VSMCs) to 6 (expression in more than two-thirds of all VSMCs).

Table 1. Patient characteristics

\begin{tabular}{|c|c|c|c|c|c|c|}
\hline Variable & $\begin{array}{c}\text { TAV-AoS } \\
\mathbf{n}=7\end{array}$ & $\begin{array}{c}\text { TAV-AoR } \\
\text { n=10 }\end{array}$ & $\begin{array}{c}\text { BAV-AoS } \\
\text { RCC/NCC } \\
n=8\end{array}$ & $\begin{array}{c}\text { BAV-AoS } \\
\text { RCC/LCC } \\
\text { n=14 }\end{array}$ & $\begin{array}{c}\text { BAV-AoS } \\
\text { RCC/LCC } \\
n=6\end{array}$ & $\begin{array}{c}\text { BAV-AoR } \\
\text { RCC/LCC } \\
n=8\end{array}$ \\
\hline Age (years) & $62 \pm 6$ & $58 \pm 11$ & $55 \pm 10$ & $59 \pm 7$ & $60 \pm 10$ & $49 \pm 3$ \\
\hline Male gender & $6(86 \%)$ & $8(80 \%)$ & $5(63 \%)$ & $10(71 \%)$ & $5(83 \%)$ & $6(75 \%)$ \\
\hline Proximal aorta $(\mathrm{mm})$ & $38 \pm 4$ & $50 \pm 3$ & $40 \pm 4$ & $38 \pm 4$ & $49 \pm 1$ & $55 \pm 5$ \\
\hline
\end{tabular}

TAV: Tricuspid aortic valve, BAV: Bicuspid aortic valve, AoS: aortic valve stenosis. AoR: aortic valve regurgitation 

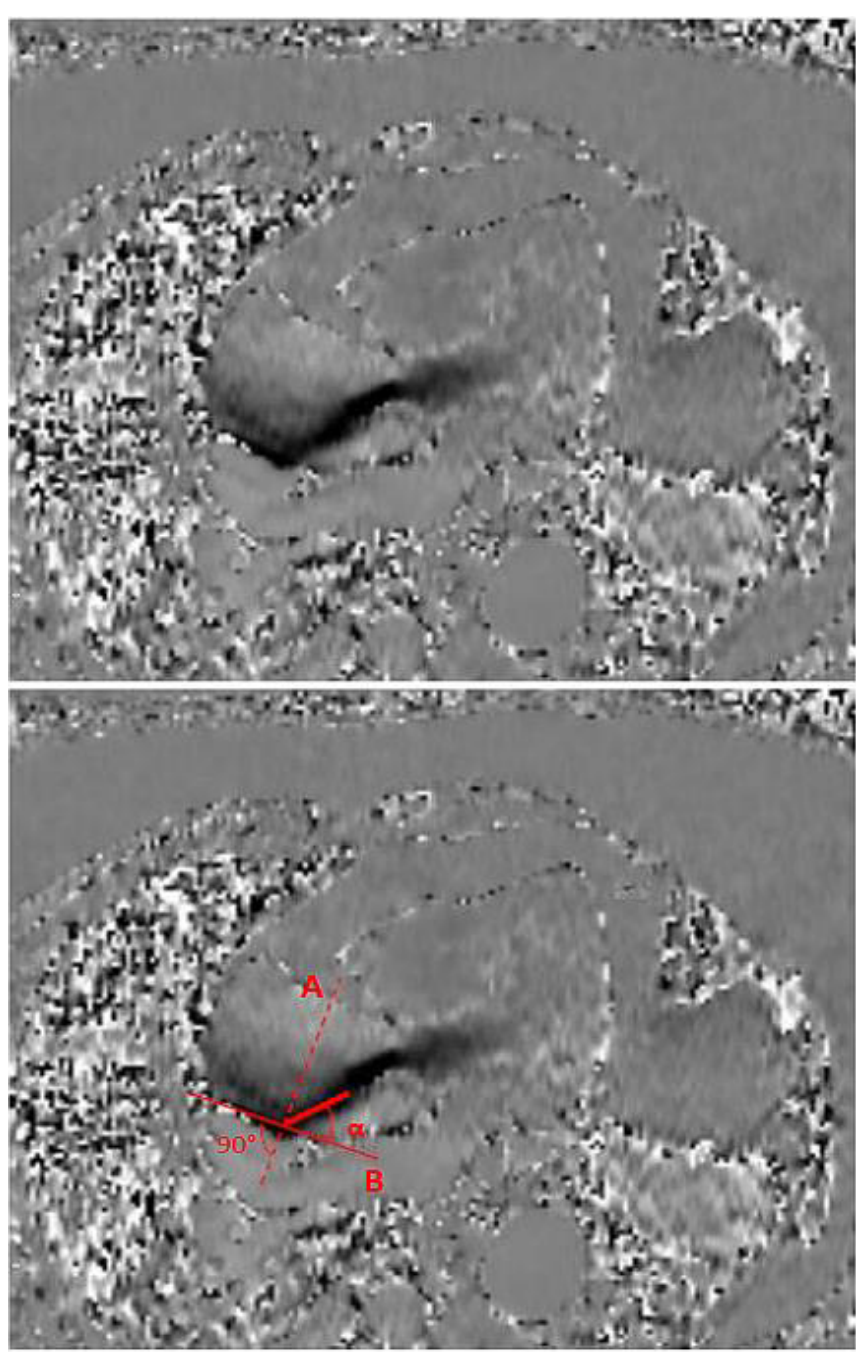

Figure 1. Figure adapted from Girdauskas et al. (29). Angle between systolic peak velocity flow jet and the aortic area in direct contact with the flow jet as identified by CMR (Flow Velocity-Encoded Phase-Contrast Imaging). (A) Cross-sectional line across the proximal aorta at the point where the systolic flow jet contacts on the aortic wall; (B) tangential line, perpendicular to line A; $\alpha=$ angle between line $\mathrm{B}$ and vector of transvalvular flow (i.e., jet aorta angle). $\mathrm{CMR}=$ cardiac magnetic resonance.

All specimens were evaluated by two independent researchers, who were blinded to the collection site of aortic specimens (i.e. jet sample versus non-jet sample).

\section{Statistical analysis}

Standard definitions were used for patient variables and outcomes. Categorical variables are expressed as percentages and continuous variables are expressed as mean $\pm \mathrm{SD}$ with range. All analyses were performed with the IBM SPSS 19.0 software (IBM corp., New York, NY, USA). For the effect of the non-jet vs jet we calculated odds-ratios between the two conditions. To accommodate the repeated, within patient (non-jet vs jet), four-category, ordered, outcomes we used Generalized Estimating Equations (GEE) to apply repeated measures ordinal logistic regression to calculate the odds ratios of the non-jet vs the jet condition adjusting for possible other predictors.

\section{Results}

Differences between non-jet bicuspid and tricuspid ascending aortic wall specimen
The described pathology score was first evaluated in the nonjet samples of BAV and TAV patients, both non- and dilated before comparing the jet to the non-jet side.

The ascending aortic wall consists of three layers: the tunica intima, media (consisting roughly of equal parts of inner, middle and outer media) and adventitia (Figure 2).

The intima was significantly thicker in all TAVs versus BAVs $(\mathrm{p}=0.004)$. Atherosclerosis in the tunica intima was more severe in the TAV versus BAV specimen ( $\mathrm{p}=0.044)$.

The media showed significantly more differentiated VSMCs $(p=0.001)$, MEMA $(p=0.018)$ (Figure $3 \mathrm{~A})$, accompanied by EFD $(\mathrm{p}=0.001)$ (Figure $3 \mathrm{~B})$ and $\mathrm{CMD}(\mathrm{p}=0.001)$ (Figure $3 \mathrm{C})$ in the dilated TAVs. Adventitial inflammation was not apparent in both BAV and TAV samples. The described differences in intima and media in the dilated and non-dilated BAV and TAV population were in line with earlier studies on a different population, except for the incidence of inflammation [3].

\section{Differences between the jet and the non-jet in bicuspid and tricuspid specimen}

To study the effects of hemodynamics paired comparisons of the pathology score were performed between the jet and non-jet sides within the BAV and TAV group (Table 2). The parameters for intimal atherosclerosis, medial VSMC differentiation, EFD, MEMA and CMD differences between the jet and non-jet side remained non-significant even if corrected for aortic dilation, the raphe position and whether the aortic valve was stenotic or regurgitant. Despite having an identical pathology score in the outer and middle aortic media, some differences were noted between the jet and non-jet samples in the inner media and intima in both BAV and TAV which were not covered by the parameters studied in the pathology score. First of all, independent of the difference in intimal thickness between BAV and TAV, as described for the non-jet samples, we observed a significant increase in intimal thickening on the jet side as compared to the non-jet side in both BAV $(\mathrm{p}=0.0005)$ and TAV $(\mathrm{p}=0.0034)$ patients (Figure 4A-F, graph $3 \mathrm{G})$. The tunica media thickness, however, did not differ significantly between the jet and non-jet sample.

We further observed that the internal elastic lamella on the borderline with the inner media was more fragmented in the jet as compared to non-jet side. It fanned out making the borderline between intima and inner media fuzzy. Thus, the distance between the endothelial layer and the first marked internal elastic lamellae appears larger on the jet side in all studied specimen (Figure 4A-F). Besides an enlargement of the distance between the endothelial layer and the internal elastic lamellae, the structure of the intimal layer was also changed. The intima in the non-jet samples of all BAV and TAV was almost devoid of aSMA expressing cells (Figure 4C), whereas in the jet samples there is an obvious increase in expression of aSMA mostly in the outer intima (Figure 4F). In the inner media, opposed to an increase of aSMA in the outer intima, significant decrease in aSMA expression on the jet side in both BAV $(\mathrm{p}<0.0001)$ and TAV $(\mathrm{p}=0.0074)$ (Figure $4 \mathrm{~F}$, graph $4 \mathrm{H}$ ) was seen. The decrease in aSMA expression in the inner media was not accompanied by loss of elastic fibers or loss of nuclei in the inner media (Figure 4D,E)). The observed differences between the jet- and non-jet samples were independent of aortic dilation, the position of the raphe and whether the aortic valve was stenotic and regurgitant. 


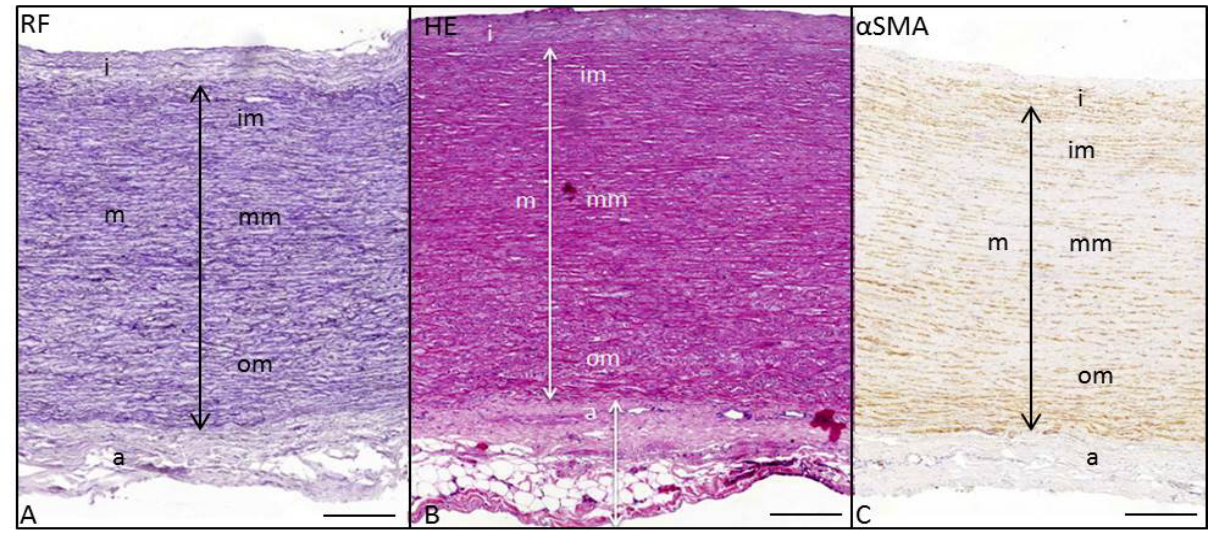

Figure 2. Transverse histologic section $(5 \mu \mathrm{m})$ of the ascending aortic wall in a non-dilated TAV (non-jet side), stained with resorcin fuchsin (A), hematoxylin eosin (B) and alpha smooth muscle actin $(\alpha \mathrm{SMA})(\mathrm{C})$. The arrows indicate the tunica intima (i), tunica media (m), tunica adventitia (a), inner media (im), middle media (mm) and outer media (om). Scale bar $100 \mu \mathrm{m}$.

Table 2 . Results

\begin{tabular}{|c|c|c|c|c|c|c|}
\hline Features & Score & $\begin{array}{r}\text { BAV-jet } \\
\text { n(\%) }\end{array}$ & $\begin{array}{c}\text { BAV-non jet } \\
\text { n(\%) }\end{array}$ & $p$-value & Odds ratio & 95\% C.I. \\
\hline \multirow[t]{4}{*}{ Atherosclerosis } & 0 & $33(91)$ & $30(91)$ & 0.371 & 1.729 & $0.521-0.5733$ \\
\hline & 2 & $1(3)$ & $1(3)$ & & & \\
\hline & 4 & $1(3)$ & $2(6)$ & & & \\
\hline & 6 & $1(3)$ & $0(0)$ & & & \\
\hline \multirow[t]{4}{*}{ SMC differentiation } & 0 & $3(9)$ & $0(0)$ & 0.395 & 1.517 & $0.581-3.962$ \\
\hline & 2 & & $28(80)$ & & $29(85)$ & \\
\hline & 4 & & $4(11)$ & & $5(15)$ & \\
\hline & 6 & & $0(0)$ & & $0(0)$ & \\
\hline \multirow[t]{4}{*}{ EFD } & 0 & $31(86)$ & $29(88)$ & 0.744 & 0.920 & $0.558-1.517$ \\
\hline & 2 & $4(11)$ & $3(9)$ & & & \\
\hline & 4 & $1(3)$ & $1(3)$ & & & \\
\hline & 6 & $0(0)$ & $0(0)$ & & & \\
\hline \multirow[t]{4}{*}{ MEMA } & 0 & $7(20)$ & $5(15)$ & 0.453 & 1.246 & $0.702-2.210$ \\
\hline & 2 & & $26(74)$ & & $27(82)$ & \\
\hline & 4 & & $2(6)$ & & $1(3)$ & \\
\hline & 6 & & $0(0)$ & & $0(0)$ & \\
\hline \multirow[t]{4}{*}{ CMD } & 0 & $29(81)$ & $32(94)$ & 0.051 & 0.235 & $0.059-1.041$ \\
\hline & 2 & $7(19)$ & $2(6)$ & & & \\
\hline & 4 & $0(0)$ & $0(0)$ & & & \\
\hline & 6 & $0(0)$ & $0(0)$ & & & \\
\hline \multirow[t]{4}{*}{ Atherosclerosis } & 0 & $8(57)$ & $11(69)$ & 0.569 & 0.828 & $0.433-1.585$ \\
\hline & 2 & $4(29)$ & $4(25)$ & & & \\
\hline & 4 & $1(7)$ & $1(6)$ & & & \\
\hline & 6 & $1(7)$ & $0(0)$ & & & \\
\hline \multirow[t]{4}{*}{ SMC differentiation } & 0 & $1(7)$ & $0(0)$ & 0.586 & 1.007 & $0.982-1.034$ \\
\hline & 2 & $1(7)$ & $2(13)$ & & & \\
\hline & 4 & $5(33)$ & $6(40)$ & & & \\
\hline & 6 & $8(53)$ & $7(47)$ & & & \\
\hline \multirow[t]{4}{*}{ EFD } & 0 & $7(44)$ & $6(38)$ & 0.672 & 1.190 & $0.532-2.660$ \\
\hline & 2 & $4(25)$ & $6(38)$ & & & \\
\hline & 4 & $4(25)$ & $3(19)$ & & & \\
\hline & 6 & $1(6)$ & $1(6)$ & & & \\
\hline \multirow[t]{4}{*}{ MEMA } & 0 & $1(8)$ & $1(7)$ & 0.514 & 0.715 & $0.262-1.957$ \\
\hline & 2 & $5(39)$ & $8(53)$ & & & \\
\hline & 4 & $7(54)$ & $6(40)$ & & & \\
\hline & 6 & $0(0)$ & $0(0)$ & & & \\
\hline \multirow[t]{4}{*}{ CMD } & 0 & $6(40)$ & $5(33)$ & 0.987 & 0.994 & $0.467-2.113$ \\
\hline & 2 & $3(20)$ & $5(33)$ & & & \\
\hline & 4 & $4(27)$ & $3(20)$ & & & \\
\hline & 6 & $2(13)$ & $2(13)$ & & & \\
\hline
\end{tabular}




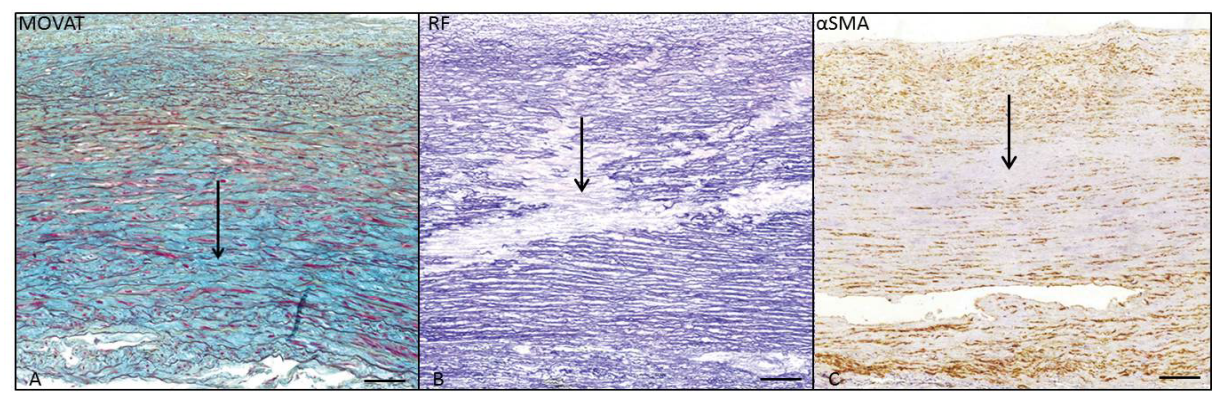

Figure 3. Transverse histologic section of the middle media of the ascending aortic wall, in a dilated TAV (non-jet side), stained with MOVAT pentachrome staining (A), resorcin fuchsin (B) and alpha smooth muscle actin ( $\alpha$ SMA) $(C)$. The arrows indicate extracellular matrix accumulation (MEMA) in 2A, elastic fiber loss and fragmentation in 2B and loss of VSMC nuclei in 2 C. Scale bar $50 \mu \mathrm{m}$.
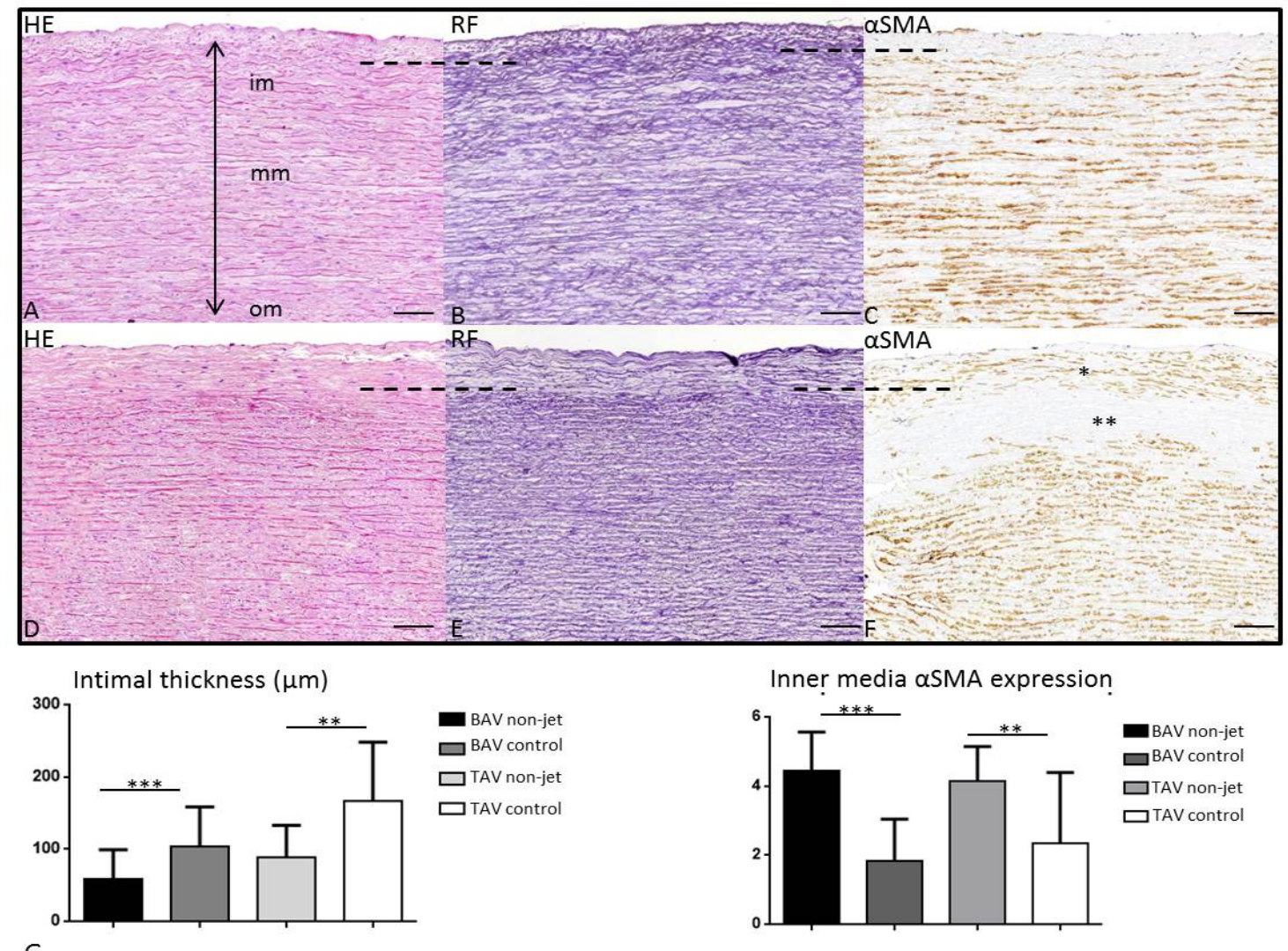

Figure 4. Transverse histologic sections $(5 \mu \mathrm{m})$ of the ascending aortic wall in a dilated BAV comparing the non-jet (A-C) and the jet side (D-F). The sections are stained for hematoxylin eosin (HE) (A,D), resorcin fuchsin (RF) (B,E) and alpha smooth muscle actin ( $\alpha$ SMA) (C,F). The borderline of intima and inner media is indicated by the dashed line, showing a significantly thicker intima in the jet as compared to the non-jet side (graph $\mathrm{G})$. At the jet side an increase in $\alpha$ SMA expression is seen in the outer thickened intima (F, indicated with *) as well as a significant decrease of $\alpha$ SMA expression in the inner media (F, indicated with **), graph H), which is however not accompanied by loss of elastic lamellae (E) or VSMC nuclei (D) in the inner media. Scale bar $100 \mu \mathrm{m}$.

\section{Discussion}

Bicuspidy is the most common cardiac malformation, with an increased risk for aortic complications as aortic dilation and dissection. In search of factors leading to an increased vulnerability for complications both genetic and hemodynamic explanations have been put forward. In this study we aimed to address the hemodynamic theory by studying the effect of flow/shear stress on the architecture of the ascending aortic wall. A pathology score was developed focusing on seven histopathology parameters: intimal architecture, intimal atherosclerosis, medial VSMC differentiation, EFD, MEMA and CMD and adventitial inflammation. The results show that in the current patient population we could confirm earlier described differences between the BAV and TAV patients [3,7] to which we could now add that this was consistent for the non-jet and jet side of the aortic wall. Between the paired jet and non-jet samples, histopathology features described in the pathology score were similar in the adventitia, and media in both BAV and TAV. Even after correcting for factors as raphe position for BAV, aortic dilation and aortic valve dysfunction.

The parameters used for the pathology score did not allow us to detect differences between the jet and non-jet group in the middle and outer media. However, jet related changes were observed in the inner media and intima. 
A significant increase in intimal thickness was noted between the BAV and TAV jet samples versus the paired non-jet samples. Almost a complete loss of VSMC actin expression in the inner media was found. Supported by literature data [19-23] we propose a flow related phenomenon. Human VSMCs are highly specialized and have the unique ability to undergo a phenotypic switch from a quiescent contractile state to a more proliferative, synthetic state [19] accompanied by loss of VSMC actin. Dedifferentiation of VSMCs from a contractile to synthetic state can occur in response to changes in local environmental cues such as hemodynamic circumstances that normally regulate the phenotype of VSMCs [20,21,23].

The synthetic VSMCs proliferate, migrate and produce ECM components [19]. Flow mediated dedifferentiation of VSMCs has been described in patients with untreated transposition of the great arteries The pulmonary artery, which was exposed to increased shear stress from the systemic circulation, also showed loss of VSMC actin expression in the inner and middle media, with an increase in the distance between the endothelial cell layer and the internal elastic lamella [22]. During development of intimal thickening in the human ductus arteriosus, a vessel subject to marked hemodynaic stress, a phenotypic aSMA poor population of VSMCs was observed in the intimal cushion [24]. Dedifferentiation of VSMCs was also detected in the neo-intima of growing coronary collaterals [25]. In our study the inner media in the non-jet samples showed expression of aSMA whereas in the jet samples the expression is lost and an increase in expression is seen in the intimal layer, which could be compared to the development of a neointima in coronary collateral vessels as described by Schaper [25]. The loss of SMA expression in the inner media and without loss of VSMC nuclei is also a support for for a phenotypic switch to a non-contractile state of the VSMCs, which generally is a prerequisite for migration and proliferation $[19,26]$. We postulate, therefore, that resident contractile VSMCs in the inner media dedifferentiate under influence of jet flow and migrate to the intimal layer producing ECM promoting the further influx of cells leading to thickening of the intimal. Migration of synthetic VSMCs leading to intimal thickening has extensively been studied in the pathogenesis of atherosclerosis, confirming that a large proportion of experimental intimal thickening arises from VSMCs $[19,27,28]$.

Normal functioning BAVs have been associated with disturbed flow patterns in the ascending aorta, with regional increases in jet impact $[13,14]$. Functional phenotype of the BAV (stenosis or regurgitation) correlates significantly with the severity of bicuspid aortopathy $[29,30]$. In our study we could not reproduce these differences in pathology score features in the middle media, even if corrected for aortic stenosis/ regurgitation, ascending aortic dilation and raphe position.

Importantly, $20-40 \%$ of BAV patients do not develop aortic complications, implicating that the BAV population is heterogeneous in terms of susceptibility for aortopathy. The described jet mediated changes in the inner layers of the aortic wall are seen in all BAV patients and In our study. The observed histopathology of intima and inner media accompanied by a VSMC phenotypic switch is a more general vascular pathology phenomenon most probably related to altered hemodynamics and shear stress as observed in many vascular remodeling situations [19-26].

In conclusion, the characteristic features of BAV aortopathy do not seem to be mediated by the hemodynamic factors related to the altered flow patterns specific for BAV. The jet related intimal thickening is observed in both TAV and BAV patients independent of dilation. The aortopathy found in BAV is therefore most probably primarly linked to a developmental, perhaps genetic, disturbance.

\section{Acknowledgement}

None

\section{Funding statement}

None

\section{Conflict of interest}

None

\section{References}

1. Roberts WC, Vowels TJ, Ko JM (2012) Natural history of adults with congenitally malformed aortic valves (unicuspid or bicuspid). Medicine (Baltimore) 91: 287-308. [Crossref]

2. Verma S, Siu SC (2014) Aortic dilatation in patients with bicuspid aortic valve. $N$ Engl J Med 370: 1920-1929. [Crossref]

3. Grewal N, Gittenberger-de Groot AC, Poelmann RE, Klautz RJ, Lindeman JH, et al. (2014) Ascending aorta dilation in association with bicuspid aortic valve: a maturation defect of the aortic wall. J Thorac Cardiovasc Surg 148(4): 1583-90. [Crossref]

4. Grewal N, Gittenberger-de Groot AC, DeRuiter MC, Klautz RJ, Poelmann RE, et al. (2014) Bicuspid aortic valve: phosphorylation of c-Kit and downstream targets are prognostic for future aortopathy. Eur J Cardiothorac Surg 46(5): 831-9. [Crossref]

5. Fedak PW, de Sa MP, Verma S, Nili N, Kazemian P, et al. (2003) Vascular matrix remodeling in patients with bicuspid aortic valve malformations: implications for aortic dilatation. J Thorac Cardiovasc Surg 126(3): 797-806. [Crossref]

6. Pasta S, Phillippi JA, Gleason TG, Vorp DA (2012) Effect of aneurysm on the mechanical dissection properties of the human ascending thoracic aorta. $J$ Thorac Cardiovasc Surg 143(2): 460-7. [Crossref]

7. Matthias Bechtel JF, Noack F, Sayk F, Erasmi AW, Bartels C, et al. (2003) Histopathological grading of ascending aortic aneurysm: comparison of patients with bicuspid versus tricuspid aortic valve. J Heart Valve Dis 12(1): 54-9. [Crossref]

8. Hiratzka LF, Creager MA, Isselbacher EM, Svensson LG, Nishimura RA, et al. (2016) Surgery for Aortic Dilatation in Patients With Bicuspid Aortic Valves: A Statement of Clarification From the American College of Cardiology/American Heart Association Task Force on Clinical Practice Guidelines. Circulation 67(6): 724-31. [Crossref]

9. Della Corte A, Bancone C, Quarto C, Dialetto G, Covino FE, et al. (2007) Predictors of ascending aortic dilatation with bicuspid aortic valve: a wide spectrum of disease expression. Eur J Cardiothorac Surg 31(3): 397-404; discussion -5. [Crossref]

10. Girdauskas E, Borger MA, Secknus MA, Girdauskas G, Kuntze T (2011) Is aortopathy in bicuspid aortic valve disease a congenital defect or a result of abnormal hemodynamics? A critical reappraisal of a one-sided argument. Eur J Cardiothorac Surg 2011;39(6): 809-14. [Crossref]

11. Cotrufo M, Della Corte A (2009) The association of bicuspid aortic valve disease with asymmetric dilatation of the tubular ascending aorta: identification of a definite syndrome. J Cardiovasc Med (Hagerstown) 10(4): 291-7. [Crossref]

12. Hope MD, Hope TA, Meadows AK, Ordovas KG, Urbania TH, et al. (2010) Bicuspid aortic valve: four-dimensional MR evaluation of ascending aortic systolic flow patterns. Radiology 255: 53-61. [Crossref]

13. Barker AJ, Markl M, Burk J, Lorenz R, Bock J, et al. (2012) Bicuspid aortic valve is associated with altered wall shear stress in the ascending aorta. Circ Cardiovasc Imaging 5(4): 457-66. [Crossref]

14. Guzzardi DG, Barker AJ, van Ooij P, Malaisrie SC, Puthumana JJ, et al. (2015) ValveRelated Hemodynamics Mediate Human Bicuspid Aortopathy: Insights From Wall Shear Stress Mapping. J Am Coll Cardiol 66(8): 892-900. [Crossref]

15. Baumgartner H, Hung J, Bermejo J, Chambers JB, Evangelista A, et al. (2009) Echocardiographic assessment of valve stenosis: EAE/ASE recommendations for clinical practice. J Am Soc Echocardiogr 10(1): 1-25. [Crossref]

16. Girdauskas E, Rouman M, Disha K, Scholle T, Fey B, et al. (2014) Correlation between systolic transvalvular flow and proximal aortic wall changes in bicuspid aortic valve stenosis. Eur J Cardiothorac Surg 46(2): 234-9. [Crossref]

17. van Dijk RA, Virmani R, von der Thusen JH, Schaapherder AF, Lindeman JH (2010) The natural history of aortic atherosclerosis: a systematic histopathological evaluation of the peri-renal region. Atherosclerosis 210(1): 100-6. [Crossref] 
18. Halushka MK, Angelini A, Bartoloni G, Basso C, Batoroeva L, et al. (2016) Consensus statement on surgical pathology of the aorta from the Society for Cardiovascular Pathology and the Association For European Cardiovascular Pathology: II. Noninflammatory degenerative diseases - nomenclature and diagnostic criteria. Cardiovasc Pathol 25(3): 247-57. [Crossref]

19. Owens GK, Kumar MS, Wamhoff BR (2004) Molecular regulation of vascular smooth muscle cell differentiation in development and disease. Physiol rev 84(3): 767-801. [Crossref]

20. Owens GK (1996) Role of mechanical strain in regulation of differentiation of vascular smooth muscle cells. Circulation res 79(5): 1054-5.

21. Langille BL (1996) Arterial remodeling: relation to hemodynamics. Can J Physiol Pharmacol 74: 834-841. [Crossref]

22. Lalezari S, Hazekamp MG, Bartelings MM, Schoof PH, Gittenberger-De Groot AC (2003) Pulmonary artery remodeling in transposition of the great arteries: relevance for neoaortic root dilatation. J Thorac Cardiovasc Surg 126(4): 1053-60. [Crossref]

23. Chamley-Campbell J, Campbell GR, Ross R (1979) The smooth muscle cell in culture. Physiol Rev 59: 1-61. [Crossref]
24. Slomp J, Gittenberger-de Groot AC, Glukhova MA, Conny van Munsteren J, Kockx MM, et al. (1997) Differentiation, dedifferentiation, and apoptosis of smooth muscle cells during the development of the human ductus arteriosus. Arterioscler Thromb Vasc Biol 17(5): 1003-9. [Crossref]

25. Wolf C, Cai WJ, Vosschulte R, Koltai S, Mousavipour D, et al. (1998) Vascular remodeling and altered protein expression during growth of coronary collateral arteries. J Mol Cell Cardiol 30(11): 2291-305. [Crossref]

26. Campbell JH, Campbell GR (2012) Smooth muscle phenotypic modulation--a personal experience. Arterioscler Thromb Vasc Biol 2012;32(8): 1784-9. [Crossref]

27. Chaabane C, Coen M, Bochaton-Piallat ML (2014) Smooth muscle cell phenotypic switch: implications for foam cell formation. Curr Opin Lipidol 25(5): 374-9. [Crossref]

28. Ross R, Glomset JA (1976) The pathogenesis of atherosclerosis (first of two parts). $N$ Engl J Med 295: 369-377. [Crossref]

29. Girdauskas E, Rouman M, Disha K, Fey B, Dubslaff G, et al. (2016) Functional Aortic Root Parameters and Expression of Aortopathy in Bicuspid Versus Tricuspid Aortic Valve Stenosis. J Am Coll Cardiol 67(15): 1786-96. [Crossref]

30. Girdauskas E, Rouman M, Disha K, Fey B, Dubslaff G, et al. (2016) Morphologic and Functional Markers of Aortopathy in Patients With Bicuspid Aortic Valve Insufficiency Versus Stenosis. The Ann Thorac Surg [Crossref]

Copyright: $\odot 2017$ Grewal N. This is an open-access article distributed under the terms of the Creative Commons Attribution License, which permits unrestricted use, distribution, and reproduction in any medium, provided the original author and source are credited. 